\title{
ON THE STABILITY OF SOME FLOWS OF AN IDEAL FLUID WITH FREE SURFACES*
}

\author{
BY \\ J. L. FOX AND G. W. MORGAN \\ Brown University
}

1. Introduction. Steady state plane flows of an incompressible inviscid fluid with free surfaces were originally studied by Helmholtz [2] and Kirchoff [3], and have since been thoroughly reported in the literature. Their work was an attempt to improve the classical solutions of flow around sharp corners which are physically unacceptable because they involve infinite velocities at the corners. Helmholtz and Kirchoff reasoned that as the velocity becomes large, the pressure in the fluid decreases to the value at which the fluid goes over into the vapor state. This gives rise to a so-called cavitated region bounded by a "free surface" over which the pressure is assumed to be maintained constant and uniform.

One also deals with steady free surface flows in the case of jets flowing in an ambient constant pressure atmosphere. Several examples of such problems are treated by MilneThompson [4].

Very little work has been done in the past concerning time dependent flows with free surfaces. Lord Kelvin [5] discussed the vibrations of a hollow columnar vortex flow. Some unsteady free surface flows under the influence of external forces, such as gravity waves in water, are discussed in Lamb [6] and the investigation there is extended to include the effects of surface tension and viscosity.

Recently Ablow and Hayes [1] developed a theory of the small perturbations of the two-dimensional flow of a perfect fluid in the presence of a free surface without external forces. They then used their theory to study two specific problems, namely the flow around a hollow vortex and the flow through a Borda mouthpiece.

The present investigation will concern itself with an extension of the work of Ablow and Hayes to some free surface flows of jets as well as to a number of generalizations of problems treated in [1]. Our primary concern will be to obtain information concerning the stability of these flows.

2. Resume of basic theory. The basic theory underlying the methods used in this report has been discussed in detail in the work of Ablow and Hayes [1]. For the sake of convenience however, a brief outline of the important results will be given below.

A. Assumptions. We shall be dealing with perturbations of steady state flows which do not fill the entire plane. They can be conveniently divided into three categories: (1) flows which are cavitated due to the fact that there is a minimum pressure the fluid can sustain; (2) jet type flows; (3) flows which are a combination of (1) and (2). In all cases there exist in the steady flow free surfaces along which the pressure remains constant and uniform.

The fluid is assumed homogeneous, incompressible and inviscid. Both the steady and perturbed states are assumed to be irrotational and two-dimensional.

${ }^{*}$ Received March 2, 1953. This paper is a condensed version of a technical report, bearing the same title, prepared for the Office of Naval Research under Contract N7onr-35807 (NR-062-090) with Brown University. 
All quantities are written in non-dimensional form through the use of a characteristic length, pressure and velocity in such a manner as to make the steady state velocity along the free surface of unit magnitude.

Under the assumptions made the flows must satisfy Bernoulli's equation in the form

$$
\varphi+\rho q^{2}+\rho \varphi \cdot=C(t),
$$

where $p$ is the pressure, $\rho$ the density, $q$ the velocity, $\varphi$ the velocity potential and $C(t)$ is a function of time alone. The dot indicates partial differentiation with respect to time.

Furthermore, we can introduce a complex potential $f$ and a complex velocity $w$ such that

$$
w=\frac{d f}{d z}
$$

where $w=u-i v$ and $u$ and $v$ are the Cartesian velocity components in the $z=x+i y$ plane.

B. Basic flow. The basic steady flow satisfies the steady form of (2.1)

$$
p_{0}+\frac{1}{2} \rho q_{0}^{2}=\text { const., }
$$

where the use of a zero subscript denotes the basic steady flow. Since

$$
w_{0}=\frac{d f_{0}}{d z_{0}} \quad \text { and } \quad q_{0}=\left|w_{0}\right|
$$

we can write (2.3) as

$$
p_{0}+\frac{1}{2} \rho w_{0} w_{0}^{*}=\text { const., }
$$

where the star indicates the operation of taking the complex conjugate*.

C. Perturbation relations. We shall now give the steady state basic flow a small perturbation in terms of a small real parameter $\epsilon$ in the form

$$
\begin{aligned}
z & =z_{0}+\epsilon z_{1}\left(z_{0}, t\right), \\
f(z, t) & =f_{0}\left(z_{0}\right)+\epsilon f_{1}\left(z_{0}, t\right), \\
w(z, t) & =w_{0}\left(z_{0}\right)+\epsilon w_{1}\left(z_{0}, t\right), \\
p(z, t) & =p_{0}\left(z_{0}\right)+\epsilon p_{1}\left(z_{0}, t\right),
\end{aligned}
$$

where $f$ and $w$ are analytic functions of $z_{0}$. All subsequent relations will be linearized by neglecting terms of order $\epsilon^{2}$ and higher. Hence all perturbations are small perturbations in that they are correct only to first order in $\epsilon$. It is convenient to perturb the independent variable $z_{0}$, although the perturbations in $f, w$ and $p$ are given in terms of the fixed point $z_{0}$.

The perturbations given above are not independent since we can derive the following relations from (2.1) and (2.2)

$$
\begin{gathered}
w_{0}^{2} z_{1}^{\prime}+w_{1}=w_{0} f_{1}^{\prime}, \\
p_{1}+\operatorname{Re}\left[w_{1} w_{0}^{*}+f_{i}-w_{0} z_{1}\right]=0,
\end{gathered}
$$

*The star is used instead of the usual bar, for typographical reasons. 
where the prime indicates partial differentiation with respect to $f_{0}$, and Re denotes the real part. Thus we see that only two of the four perturbations are independent.

We note that, when properly chosen, two different sets of perturbations, e.g., $\left(z_{3}, f_{3}\right)$ and $\left(z_{4}, f_{4}\right)$ may represent the same physical perturbation. Their difference, namely $z_{\mathrm{I}}=z_{3}-z_{4}, f_{\mathrm{I}}=f_{3}-f_{4}$, will leave the flow unchanged and the perturbation $\left(z_{1}, f_{\mathrm{I}}\right)$ will be called an invariant perturbation.

We define a stationary perturbation $\left(z_{2}, f_{2}\right)$ to be one in which any given physical perturbation is evaluated at a fixed point $z_{0}$ of the basic flow, i.e., one for which the space variable is not perturbed $\left(z_{2} \equiv 0\right)$. We can now find, corresponding to a given perturbation $\left(z_{1}, f_{1}\right)$, a unique stationary form by superposing on $\left(z_{1}, f_{1}\right)$ the invariant perturbation $z_{1}=-z_{1}$.

Using (2.4) and (2.5) we can derive the following relations between the stationary perturbations $f_{2}, w_{2}$ and $p_{2}$ :

$$
\begin{gathered}
w_{2}=w_{0} f_{2}^{\prime}, \\
p_{2}=-\rho \operatorname{Re}\left[w_{0} w_{0}^{*} f_{2}^{\prime}+f_{2}^{\prime}\right] .
\end{gathered}
$$

In this formulation there is only one independent perturbation quantity, say $f_{2}$, restricted only by the condition that it be admissible under the boundary conditions of the problem.

In subsequent work, for the sake of compactness, we shall not change the name of a function after a change of independent variable, e.g., we shall write

$$
f\left(z_{0}\right)=f\left[w_{0}\left(z_{0}\right)\right]=f\left(w_{0}\right) .
$$

D. Free surface condition. There are two conditions that must hold on the free surface. First, the free surface pressure remains constant, and second, a particle originally on the free surface remains on the free surface in the perturbed state. From (2.5) we see that we can satisfy the first condition by demanding that

$$
\operatorname{Re}\left[f_{2}^{\prime}+f_{2}^{\prime}+w_{0}^{\prime} z_{1}\right]=0
$$

where we have used the relation between $f_{1}$ and its stationary form $f_{2}$

$$
f_{2}=f_{1}-w_{0} z_{1} \text {. }
$$

The second condition can be shown to imply that

$$
\operatorname{Im}\left[f_{2}^{\prime}+\left(w_{0} z_{1}\right)^{\prime}+\left(w_{0} z_{1}\right)^{\cdot}\right]=0
$$

where Im denotes the imaginary part.

We can satisfy (2.8) identically if we set the expression in the bracket equal to $i \chi\left(z_{0}, t\right)$, where the function $\chi$ is real on the free surface and otherwise arbitrary. Solving for $z_{1}$ to obtain the perturbation in the space co-ordinate which takes the basic flow free surface into the perturbed free surface, we have

$$
z_{1}=\frac{1}{w_{0}^{\prime}}\left\{i \chi-D\left[f_{2}\right]\right\}
$$

where the operator $D[]=\partial[\quad] / \partial f_{0}+\partial[\quad] / \partial t$. Now substituting for $z_{1}$ from (2.10) in (2.9) we find, after some reduction, that the free surface boundary condition is

$$
\operatorname{Im}\left\{D\left[f_{2}-\omega D\left[f_{2}\right]\right]-f_{2}^{\cdot}\right\}=0
$$

where $\omega=w_{0} / w_{0}^{\prime}$. 
Adopting the notation

$$
H \equiv L\left[f_{2}\right] \equiv D\left[f_{2}-\omega D\left[f_{2}\right]\right]-f_{2}^{*}
$$

(2.11) becomes with $w_{0}$ as the independent variable

$$
H\left(w_{0}\right)=\left(H\left(w_{0}\right)\right)^{*} \quad \text { on } \quad w_{0} w_{0}^{*}=1 .
$$

E. Symmetry considerations. In cases where the basic flow has a velocity distribution which is a symmetric function of $z_{0}$ (symmetric basic flow) certain simplifications can be made in the problem. In functional notation a symmetric function $F(v)$ satisfies

$$
F^{*}(v)=\left(F^{*}\left(v^{*}\right)\right)^{*}
$$

while an antisymmetric function satisfies

$$
F^{a}(v)=-\left(F^{a}\left(v^{*}\right)\right)^{*}
$$

We can combine both relations in a convenient notation

$$
F^{s a}(v)= \pm\left(F^{s a}\left(v^{*}\right)\right)^{*}
$$

where the first superscript corresponds to the upper sign, and the second superscript to the lower sign.

Certain operations performed on a symmetric or antisymmetric function preserve these properties. It can be shown, for example, that the operations of differentiation and integration are symmetry preserving, i.e., the symmetry or antisymmetry of the function remains unchanged. Also, if

$$
F^{s a}(v)= \pm\left(F^{s a}\left(v^{*}\right)\right)^{*}
$$

and we transform to a new variable $u$ such that

$$
u(v)=\left(u\left(v^{*}\right)\right)^{*}
$$

then

$$
F^{* a}(u)= \pm\left(F^{s a}\left(u^{*}\right)\right)^{*}
$$

i.e., the function retains its symmetry or antisymmetry properties in the $u$ plane.

The important consequence of these considerations is embodied in a theorem which will be stated here without proof (for proof see [1, pp. 26 et seq.]).

Symmetry Theorem: If the basic flow is symmetric, any perturbation can be represented as the sum of a symmetric and an antisymmetric perturbation each of which satisfies all boundary conditions and so is an admissible perturbation in its own right.

$F$. Separation of time dependence. At this point in the development of the theory, the only restriction placed on the perturbation in the potential is that it shall satisfy all applicable boundary conditions. We shall attack the problem by assuming solutions of the form

$$
f_{2}=G_{1}\left(w_{0}\right) e^{\lambda t}+G_{2}\left(w_{0}\right) e^{\lambda * t} .
$$

We anticipate that this choice of time dependence will lead to an eigenvalue problem for the determination of the functions $G_{1}$ and $G_{2}$. We expect to find that the boundary conditions can be satisfied only for certain specific values of $\lambda$ and that a general solution will be a sum of all such elementary forms of $f_{2}$. Our primary concern will be to determine 
the magnitude of the real part of all admissible $\lambda$ since this will indicate the stability of the flow. We shall have

$$
\begin{array}{ll}
\text { unstable perturbations for } & \operatorname{Re}\{\lambda\}>0, \\
\text { neutrally stable perturbations for } & \operatorname{Re}\{\lambda\}=0, \\
\text { stable perturbations for } & \operatorname{Re}\{\lambda\}<0 .
\end{array}
$$

It might seem sufficient to assume $f_{2}$ in the form

$$
f_{2}=G\left(w_{0}\right) e^{\lambda t}
$$

since if $\lambda$ and $\lambda^{*}$ were both eigenvalues, both would be found among the admissible values of $\lambda$. The form (2.14) has been chosen because it is found that the elementary form (2.14a) is not capable of satisfying all the boundary conditions, whereas the form (2.14) can represent an admissible perturbation.

Substitution of the form for $f_{2}$ from (2.14) in our previous expression (2.12) for the operator $H$ gives

$$
H\left(w_{0}\right)=L_{\lambda}\left[G_{1}\right] e^{\lambda t}+L_{\lambda}^{*}\left[G_{2}\right] e^{\lambda * t},
$$

where

$$
L_{\lambda}[G]=\frac{w_{0}^{2}}{\omega} \frac{d^{2} G}{d w_{0}^{2}}+2 \lambda w_{0} \frac{d G}{d w_{0}}+\lambda G\left(\omega^{\prime}+\lambda \omega\right) .
$$

Our free surface boundary condition (2.13) becomes

$$
L_{\lambda}\left[G_{1}\left(w_{0}\right)\right]=\left(L_{\lambda^{*}}\left[G_{2}\left(w_{0}\right)\right]\right)^{*} .
$$

In the event that the basic flow is symmetric we can decompose $f_{2}$ into symmetric and antisymmetric components and write

$$
f_{2}=f_{2}^{s}+f_{2}^{a},
$$

where

$$
f_{2}^{s a}=G_{1}^{s a} e^{\lambda t}+G_{2}^{s a} e^{\lambda * t}
$$

and we can show that

$$
G_{2}^{s a}\left(w_{0}\right)= \pm\left(G_{1}^{s a}\left(w_{0}^{*}\right)\right)^{*} .
$$

With (2.19) we can eliminate $G_{2}$ from our free surface boundary condition (2.17) which then becomes

$$
L_{\lambda}\left[G_{1}^{s a}\left(w_{0}\right)\right]= \pm L_{\lambda}\left[G_{1}^{s a}\left(\frac{1}{w}\right)\right] .
$$

This condition is to be applied on the free surface $w_{0} w_{0}^{*}=1$ or $w_{0}^{*}=1 / w_{0}$. We can use analytic continuation, however, and demand that it hold over the entire $w_{0}$ plane.

The remainder of this paper will be devoted to solving the perturbation equation (either (2.17) or (2.20) for several types of problems. The analysis gives rise to relations which are exceptionally long and cumbersome. Due to limitations of space, only the essential features of the solutions will be presented here. For further details the reader may refer to the report mentioned in the footnote to the title. The subscript 0 in $w_{0}$, which denotes the basic flow, will be dropped in the work that follows. 
3. Jet impinging on a finite plate. One of the flows which the authors intended to study was that which results when a plate of finite width is placed at right angles to an infinite stream which is uniform at infinity and which has an infinite cavity bounded by constant pressure surfaces downstream of the plate, (the Helmholtz plate problem). Since the velocity is assumed to be the same no matter how one approaches infinity, the points corresponding to upstream and downstream infinity in the physical plane map into one and the same point in the hodograph $\left(w_{0}\right)$ plane. In the analysis of the perturbed flow one is then confronted with the problem of applying boundary conditions pertaining to different regions in the physical plane at a single point in the hodograph plane. Mathematically the result of this is that the perturbation quantities exhibit a very irregular behavior at this point; it is found, in fact, that one is led to a differential equation. which has an irregular singular point there.

To overcome this difficulty an attempt was made to differentiate between upstream and downstream infinity by artificially separating slightly the source and sink representing these points in the hodograph plane. After applying the boundary conditions the points would be made to coalesce and it was hoped that the results so obtained would be the same as if the original problem had been solved directly.

In the course of this study it was realized that the separation of upstream and downstream infinity could be accomplished in a more direct and rigorous manner by considering the Helmholtz plate as the limiting case of a related and much more general physical problem, namely that of a jet of finite width which impinges on a finite plate and is thereby divided into two jets which diverge from the edges of the plate and tend to become straight jets inclined at $\pm \theta$ to the horizontal as they approach downstream

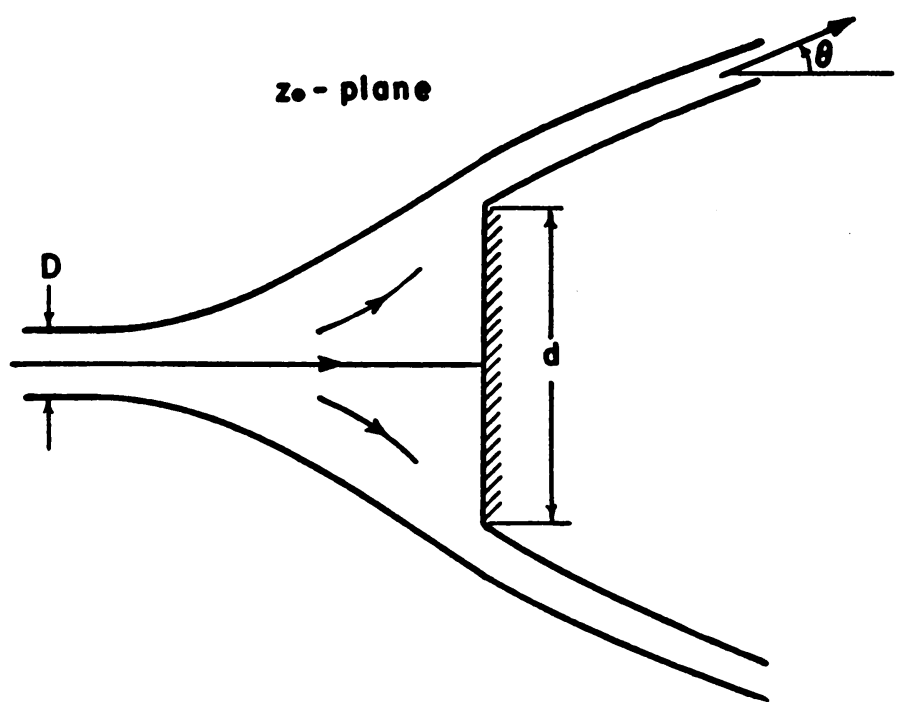

Fig. 1.

infinity (see Fig. 1). The limiting case of infinite jet width then constitutes the proper mathematical formulation of the Helmholtz plate problem.

A. Basic flow equations. We begin by writing the potential of the basic flow in the hodograph plane (see Fig. 2). This flow may be thought of as arising from the presence 
of unit sources at $w= \pm 1$ and sinks of strength one-half on the unit circle at $w= \pm a$ and $\pm a^{*}$ where argument of $a=\theta$, the jet inclination at downstream infinity. The potential is then found to be

$$
f_{0}(w)=\log \frac{\left(w^{2}-1\right)^{2}}{\left(w^{2}-a^{2}\right)\left(w-a^{*^{2}}\right)} .
$$

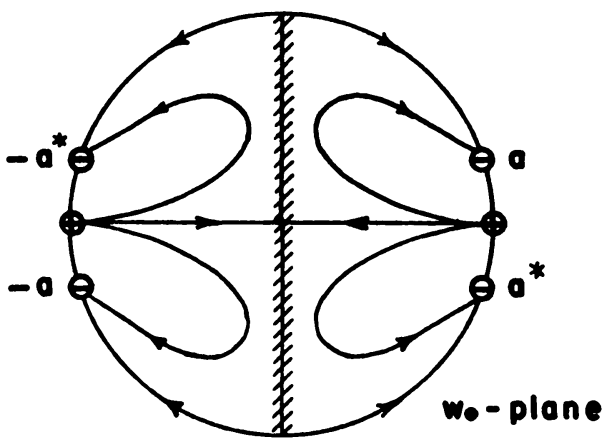

FIG. 2.

Since we are only interested in that part of the hodograph plane which corresponds to the physical flow, it is convenient to introduce a transformation $\zeta=w^{2}$ such that the right half of the unit circle in the $w$ plane goes into the entire unit circle in the $\zeta$ plane. This transformation has the advantage of reducing the number of singular points on the unit circle.

The potential in the $\zeta$ plane becomes

$$
f_{0}(\zeta)=\log \frac{(\zeta-1)^{2}}{(\zeta-b)\left(\zeta-b^{*}\right)^{\prime}}
$$

where

$$
b=a^{2} .
$$

The asymptotic inclination of the downstream jets, as characterized by a parameter $\beta=\sin \theta$, can be related to the dimensionless ratio $(d / D)$ of plate width to original jet width. This can be accomplished by considering the integral

$$
\int_{0}^{d / 2 D} d z=\frac{1}{2}\left(\frac{d}{D}\right)
$$

In terms of hodograph variables we have

$$
d z=\frac{1}{w}\left(\frac{d f_{0}}{d w}\right) d w
$$

Thus

$$
\int_{0}^{\pi / 2 D} d z=\int_{0}^{-i} \frac{1}{w}\left(\frac{d f_{0}}{d w}\right) d w=1 / 2(d / D) .
$$

Now using (3.1) for $f_{0}(w)$ which involves the unknown sink position $a$, we finally arrive at

$$
(d / D)=\pi / 2\left(1-\sqrt{1-\beta^{2}}\right)+\beta / 2 \log \left(\frac{1+\beta}{1-\beta}\right) .
$$


The limit cases $\beta=1$ and $\beta=0$ correspond to the impinging of a jet of finite width on an infinite plate, and the impinging of an infinite stream upon a finite plate, respectively. These, in turn, correspond to making the sink position $a$ approach $i$ or +1 , respectively. The steady state form of the potential, (3.1), in these limiting cases, goes over into the known form of the potential with $(d / D)=0$ or $\infty$ respectively.

$B$. Derivation of the perturbation equation. As discussed in the basic theory we try a solution of the form

$$
f_{2}=G_{1} e^{\lambda t}+G_{2} e^{\lambda * t}
$$

Our first objective is to narrow the possible choice of functions $G_{1}$ and $G_{2}$ by investigating the functional properties which $f_{2}$ must possess in order to satisfy some of the conditions of the problem.

(1) Wall streamline condition. The boundary condition on the imaginary axis (map of the plate) in the $w$ plane is that the perturbed flow have no component normal to the axis. It is convenient to transform this condition to the real axis. To do this we rotate the hodograph plane by putting $\eta=i w$. The boundary condition will now be satisfied if $w_{2} / w$ is real on $\eta$ real. Denoting differentiation with respect to $\eta$ by the subscript $\eta$ and evaluating $w_{2} / w$ we have that

$$
\frac{\eta\left(\eta^{2}+1\right)\left(\eta^{2}+b\right)\left(\eta^{2}+b^{*}\right)}{2 \eta^{2}\left\{2\left(\eta^{2}+b\right)\left(\eta^{2}+b^{*}\right)-\left(\eta^{2}+1\right)\left[\left(\eta^{2}+b\right)+\left(\eta^{2}+b^{*}\right)\right]\right.} f_{2 \eta}
$$

must be real on $\eta$ real. Since the coefficient of $f_{2 \eta}$ in the expression above is itself real for real $\eta$, we infer that $f_{2 \eta}$ must be real on $\eta$ real and hence a symmetric function of $\eta$ in $|\eta|<1$.

(2) Analyticity and symmetry considerations. Since the basic flow is everywhere regular in $|\eta|<1, f_{2 \eta}$ must also be regular in $|\eta|<1$. Upon integrating $f_{2 \eta}$ we then find $f_{2}(\eta)$ is both regular and symmetric in $|\eta|<1$, i.e.,

$$
f_{2}(\eta)=\left(f_{2}\left(\eta^{*}\right)\right)^{*} \text {. }
$$

Since the basic flow is symmetric we may decompase the perturbations into symmetric and antisymmetric components, i.e.,

$$
f_{2}=f_{2}^{s}+f_{2}^{a} \quad \text { where } \quad f_{2}^{s a}(\zeta)= \pm\left(f_{2}^{s a}\left(\zeta^{*}\right)\right)^{*} .
$$

These relations hold in the $\zeta$ and $w$ planes since the transformation from $w$ to $\zeta$ preserves symmetry.

In the $\eta$ plane $\left(\eta=i \zeta^{\frac{1}{3}}\right)$ the above becomes

$$
f_{2}^{s a}(\eta)= \pm\left(f_{2}^{s a}\left(-\eta^{*}\right)\right)^{*},
$$

where the superscripts still refer to the symmetric and antisymmetric components of $f_{2}$ referred to the $\zeta$ (or $w$ ) plane. Using the relation (3.5) we have

$$
f_{2}^{s a}(\eta)= \pm f_{2}^{s a}(-\eta) .
$$

Thus, a $\zeta$-plane symmetric or antisymmetric perturbation is represented by an even or odd function of $\eta$, respectively, and we may write

$$
f^{s}=\sum_{0}^{\infty} a_{k} \eta^{2 k}, \quad f^{a}=\sum_{0}^{\infty} b_{k} r_{i}^{2 k+1} \text {. }
$$


Since $\zeta=-\eta^{2}$ these relations transform in the $\zeta$ plane to

$$
f_{2}^{s}=F^{s}(\zeta), \quad f_{2}^{a}=\zeta^{1 / 2} F^{a}(\zeta) .
$$

where $F^{s}$ and $F^{a}$ are regular functions of $\zeta$ in $|\zeta|<1$. We can also write

$$
f_{2}^{s a}=G_{1}^{s a} e^{\lambda \ell}+G_{2}^{s a} e^{\lambda * t}
$$

where we know that, by virtue of the symmetric basic flow,

$$
G_{2}^{s a}(w)= \pm\left(G_{1}^{s a}\left(w^{*}\right)\right)^{*} .
$$

Since $\zeta=w^{2}$ is a symmetric function of $w$, the above symmetry relation also holds in the $\zeta$ plane (see $2 \mathrm{E}$ ), i.e.,

$$
G_{2}^{s a}(\zeta)= \pm\left(G_{1}^{s a}\left(\zeta^{*}\right)\right)^{*}
$$

The four functions $G_{1}^{s a}$ and $G_{2}^{s a}$ can be replaced by two functions $g^{\text {aa }}$ by the following relations which are found to be consistent with the above considerations.

$$
\begin{array}{ll}
G_{1}^{s}=g^{s}(\zeta), & G_{2}^{s}=\left(g^{s}\left(\zeta^{*}\right)\right)^{*} \\
G_{1}^{a}=g^{a}(\zeta), & G_{2}^{a}=-\left(g^{a}\left(\zeta^{*}\right)\right)^{*}
\end{array}
$$

where $g^{*}$ and $\zeta^{-\frac{1}{2}} g^{a}$ are regular functions of $\zeta$ in $|\zeta|<1$.

(3) The free surface boundary condition. For a symmetric basic flow the free surface condition in the $\zeta$ plane is

$$
L_{\lambda}\left[G_{1}^{s a}(\zeta)\right]=L_{\lambda^{*}}\left[G_{1}^{s a}\left(\frac{1}{\zeta}\right)\right],
$$

where the form of the differential operator $L_{\lambda}$ in the $\zeta$ plane is found from (2.16) and (3.1). It is not reproduced here because of space limitations.

If we substitute the forms (3.6) for $G_{1}^{s a}$ in (3.7), we arrive at the following relations

$$
\begin{aligned}
& h^{s}(\zeta)=h^{s}\left(\frac{1}{\zeta}\right) \\
& h^{a}(\zeta)=-h^{a}\left(\frac{1}{\zeta}\right)
\end{aligned}
$$

where

$$
h^{\circ a}(\zeta)=L_{\lambda}\left[g^{s a}(\zeta)\right]
$$

The form of the differential operator $L_{\lambda}$ and our knowledge of the functional behavior of $g^{*}$ and $g^{a}$ in $|\zeta|<1$ together with (3.10) show that, in $|\zeta|<1, h^{*}(\zeta)$ is a regular function of $\zeta$ and that $h^{a}(\zeta)$ behaves like $\zeta^{\xi}$ multiplied by a function with a simple pole at $\zeta=0$. For convenience we shall represent $h^{a}$ and $h^{a}$ in the following manner

$$
\begin{aligned}
& h^{a}(\zeta)=\frac{\zeta-1}{\zeta^{1 / 2}} \sum_{-\infty}^{\infty} a_{k}\left[\frac{(\zeta-1)^{2}}{(\zeta-b)\left(\zeta-b^{*}\right)}\right]^{k}, \\
& h^{\prime}(\zeta)=\sum_{-\infty}^{\infty} b_{k}\left[\frac{(\zeta-1)^{2}}{(\zeta-b)\left(\zeta-b^{*}\right)}\right]^{k} .
\end{aligned}
$$


with $a_{k}$ and $b_{k}$ unknown constants. These forms for $h^{s}$ and $h^{a}$ passess the required behavior in $|\zeta|<1$, and in addition satisfy the functional relations (3.7) termwise.

Our aim is to find functions $g^{s a}(\zeta)$ satisfying the relation (3.10) with $h^{s a}(\zeta)$ having the forms given in (3.11). To do this we regard (3.10) as an inhomogeneous differential equation for $g^{s a}(\zeta)$. We note that the inhomogeneous term $h^{s a}(\zeta)$ is known in form only and hence the solutions $g^{\text {sa }}(\zeta)$ will retain some arbitrariness which, for a given problem, ought to be determined by the initial conditions.

C. Solutions of the perturbation equation. Equation (3.10) is a second order linear differential equation of the Fuchsian type [7] with regular singular points at $\zeta=0,1, b$, $b^{*}$ and $\infty$. We can first find the complementary solution about the origin, which we can denote as $A K^{(1)}+B K^{(2)}$, by the standard methods of solution in series. Knowing the complementary solutions, the particular integral is readily found by the method of variation of parameters, viz.

$$
\text { P.I. }=K^{(1)} \int_{\zeta 。}^{\zeta} \frac{p(\zeta) K^{(2)} d \zeta}{W\left(K^{(1)}, K^{(2)}\right)}-K^{(2)} \int_{\zeta 。}^{\zeta} \frac{p(\zeta) K^{(1)}(\zeta) d \zeta}{W\left(K^{(1)}, K^{(2)}\right)}
$$

where

$$
p(\zeta)=\left[\frac{1}{\zeta(\zeta-1)}-\frac{1}{2 \zeta(\zeta-b)}-\frac{1}{2 \zeta\left(\zeta-b^{*}\right)}\right] h^{\circ a}(\zeta),
$$

and $W\left(K^{(1)}, K^{(2)}\right)$ is the Wronskian of the two solutions which can be found from the differential equation and is $\Lambda \zeta^{-3}(\zeta-1)^{-4 \lambda}(\zeta-b)^{2 \lambda}\left(\zeta-b^{*}\right)^{2 \lambda}$ where $\Lambda$ is a known constant. The point $\zeta_{0}$ is an arbitrary ordinary point of the differential equation which we shall choose as $\zeta_{0}=-1$. This solution will be valid up to the nearest singular point, i.e., within the unit circle. Since boundary conditions will have to be applied not only within, but also on the unit circle, in particular at the singular points, we must find solutions valid at these points. To do this we study the indicial equation and hence the form of the complementary solution appropriate to the point in question and then match this form of the solution with the solution around the origin by the process of analytic continuation. A new form of the particular integral may then be found. This method must be continued until we obtain solutions near all points at which boundary conditions must be applied.

To conserve space, we shall write the form of the solution around the origin only. It is

$$
g_{R}^{s a}(\zeta)=A_{R}^{s a} K^{(1)}+B_{R}^{s a} K^{(2)}+\sum_{r=0}^{\infty} E_{r}^{s a} K_{R+r}^{s a},
$$

where $A_{R}^{s a}, B_{R}^{s a}$ and $E_{r}^{s a}$ are unknown constants. The term $E_{R}^{s} K_{R+r}^{s}$ is a particular integral when $h^{\circ}(\zeta)$ in (3.12) is replaced by, (see (3.11)),

$$
h_{R+r}^{\circ}=b_{R+r}\left[\frac{(\zeta-1)^{2}}{(\zeta-b)\left(\zeta-b^{*}\right)}\right]^{R+r} \text {. }
$$

Similarly $E_{r}^{a} K_{R+r}^{a}$ is a particular integral when $h^{a}(\zeta)$ in (3.12) is replaced by

$$
h_{R+r}^{a}=\frac{\zeta-1}{\zeta^{1 / 2}} a_{R+r}\left[\frac{(\zeta-1)^{2}}{(\zeta-b)\left(\zeta-b^{*}\right)}\right]^{R+r} .
$$

The index $R$, which is an integer used to designate the solutions, is chosen such that $E_{0}^{s a}$ is the first non-zero $E^{s a}$. 
D. Application of the boundary conditions. At this stage the symmetric and antisymmetric perturbations found as solutions of the perturbation equation satisfy the wall streamline and free surface boundary conditions. We shall now investigate the restrictions to be placed on these solutions in order that they may satisfy all remaining boundary conditions. We shall first discuss the antisymmetric solutions.

(1) Anti-symmetric solutions. (a) The edges of the plate $(\zeta=-1)$.

We demand that the inner surfaces of the jets diverging from the edges of the plate continue to originate there in the perturbed state. Since $z_{1}$ is the perturbation in the space coordinate which takes the basic flow free surface into the perturbed free surface (see Eq. (2.10) in 2D), this condition will be satisfied if

$$
z_{1}=\frac{1}{w^{\prime}}\left\{i \chi-f_{2}^{\prime}-f_{2}^{\prime}\right\}=0 \quad \text { at } \quad \zeta=-1
$$

Using (3.4) and (3.6) we find this implies $g_{\zeta}^{a}(-1)=0$. The solution $g_{R}^{a}$ valid near $\zeta=-1$ will satisfy this if an equation of the form

$$
C_{1} A_{R}^{a}+C_{2} B_{R}^{a}=0
$$

is satisfied with $C_{1}$ and $C_{2}$ non-zero constants.

(b) At the point $\zeta=0$. We have all ready specified the behavior of $g^{a}(\zeta)$ in $|\zeta|<1$. We can insure that the solution $g_{R}^{a}$ possess this behavior if $\zeta^{-\frac{1}{a}} g^{a}$ is a regular function of $\zeta$ as $\zeta \rightarrow 0$.

This boundary condition can be satisfied by equating to zero the terms in the solution $g_{R}^{a}$ valid near $\zeta=0$ which do not have the required $\frac{1}{2}$ order behavior. This results in two further linear homogeneous equations to be satisfied by the unknown constants which are of the form

$$
B_{R}^{a}+\sum_{r=0}^{\infty} \alpha_{r} E_{r}^{a}=0
$$

and

$$
\sum_{r=0}^{\infty} \delta_{r} E_{r}^{a}=0
$$

where $\alpha_{r}$ and $\delta_{r}$ are non-zero constants.

(c) Downstream infinity $\left(\zeta=b\right.$ and $\left.\zeta=b^{*}\right)$. In the basic flow the jets diverging from the edges of the plate asymptotically become straight uniform jets. One can investigate the behavior of a straight jet when subjected to small disturbances using the approach described in Lamb's Hydrodynamics (Chapter IX). Such a flow is found to be neutrally stable and any disturbance is propagated downstream unchanged with the velocity of the jet. We shall demand that our perturbed flow behave like a straight jet as we approach downstream infinity. Hence, an observer moving with the asymptotic jet velocity will see no change in the velocity perturbation $w_{2}$. If we express this fact by means of the material derivative of $w_{2}$ we find that the boundary condition demands that $\lim _{\zeta \rightarrow b}(\zeta-b)^{-\lambda} g^{a}$ and $\lim _{\zeta \rightarrow 0^{*}}\left(\zeta-b^{*}\right)^{-\lambda^{*}} g^{a}$ exist.

An examination of the solutions valid about $b$ and $b^{*}$ shows them to be derivable from each other by a simple interchange of the roles of $b$ and $b^{*}$ and hence only one of the conditions has to be applied.

It is found that the terms of the complementary solution satisfy the boundary con- 
dition for any value of $\lambda$. By investigating the behavior of the integrals making up the particular integral, it can be shown that the term in $(\zeta-b)^{-\lambda} g_{R}^{a}$ with the least real part of the exponent of $(\zeta-b)$ has a non-zero coefficient and hence the boundary condition demands that the real part of the exponent be greater than zero. This implies that

$$
R+\operatorname{Re}\{\lambda\} \leq 1-r_{\max }
$$

where $r_{\max }$ is the greatest $r$ for which we must have $E_{r}^{a} \neq 0$. From (3.16) we see that, since $E_{0}^{a} \neq 0, r_{\max }$ must be at least as great as one. Hence, the inequality becomes

$$
R+\operatorname{Re}\{\lambda\} \leq 0
$$

(d) Upstream infinity $(\zeta=1)$. The basic flow originates from a source point at upstream infinity. Since we do not wish the perturbations to alter the fundamental nature of the entire flow we shall demand that the perturbation velocity $w_{2}$ and the perturbation pressure $p_{2}$ vanish at upstream infinity. These conditions will be satisfied if $(\zeta-1) g_{\zeta}^{a}$ and $g^{a}$, respectively, vanish as $\zeta \rightarrow 1$.

Since the point $\zeta=1$ is a regular singular point of the differential equation, however, our knowledge of the behavior of the solution at such a point shows that these conditions are redundant and only one, say the latter, will be applied.

We must show that in the neighborhood of $\zeta=1$ the expression for $g_{R}^{a}$ consists of terms which go to zero as $\zeta \rightarrow 1$ or that any terms which do not go to zero have a coefficient which is zero. If one examines the solution $g_{R}^{a}$ near $\zeta=1$, one finds that the dominant term from the complementary solution behaves like $(\zeta-1)^{-2 \lambda}$ while the dominant term contributed by the particular integral is either $(\zeta-1)^{-2 \lambda}$ or $(\zeta-1)^{2 R+1}$, depending upon the relative magnitude of $R$ and $\operatorname{Re}\{\lambda\}$. As yet, $R$ and $\operatorname{Re}\{\lambda\}$ are restricted only by the inequality (3.18). We can now proceed to investigate the various manners in which the boundary condition at $\zeta=1$ may be satisfied depending upon which of the following inequalities is applicable:

$$
\begin{aligned}
\text { (i) } 2 R+1<\operatorname{Re}\{-2 \lambda\}, \\
\text { (ii) } 2 R+1=\operatorname{Re}\{-2 \lambda\}, \\
\text { (iii) } 2 R+1>\operatorname{Re}\{-2 \lambda\} .
\end{aligned}
$$

Consider, for example, Case $(i)$. The term $(\zeta-1)^{2 R+1}$ has a non-zero coefficient; hence, the boundary condition demands $2 R+1>0$ which gives $R=0,1,2, \cdots$ and then the inequality shows $\operatorname{Re}\{\lambda\}<-\frac{1}{2}$. In a similar manner one finds in every case that $\operatorname{Re}\{\lambda\}<0$.

(2) Symmetric solutions. The application of the boundary conditions to the symmetric perturbations proceeds in the same manner as in the antisymmetric ones. We again find no admissible perturbations with $\operatorname{Re}\{\lambda\}>0$.

$E$. Admissible complementary solutions. One may consider the possibility of satisfying the boundary conditions with solutions containing only terms of the complementary solution. In both the antisymmetric and symmetric case one can readily show, however, that there are no such solutions capable of satisfying all the boundary conditions.

$F$. Conclusions as to the stability of the basic flow. Although we have not found a onedimensional continuum of eigenvalues $\lambda$, our present analysis has been able to restrict 
the possible values of $\lambda$ to the left half of the complex $\lambda$ plane. This shows that any admissible perturbation has $\operatorname{Re}\{\lambda\} \leq 0$ and we conclude that a jet impinging normally upon a finite plate gives a neutrally stable or stable flow configuration.

The results of this analysis are valid for any finite but non-zero ratio of plate width to jet width $(d / D)$. By making this ratio as small as we please, we can consider the flow of a jet of arbitrarily great width past a finite plate. But this is a proper physical interpretation of the Helmholtz plate problem since one never has a truly infinite stream in reality. From this point of view, the stability conclusions reached in this section apply to the Helmholtz plate problem.

On the other hand, we find a different situation if we make the ratio $(d / D)$ very large but still finite. In this case the point $\zeta=-1$ continues to be an ordinary point of the perturbation differential equation and the boundary condition $z_{1}=0$ must always be satisfied there. However, if one actually sets $d / D=\infty$, the basic flow of this section becomes that of a jet impinging on an infinite wall. Now the point $\zeta=-1$ is a regular singular point corresponding to downstream infinity and the boundary condition $z_{1}=0$ no longer applies. A reexamination of the work of the present section will show that it was the presence of this additional boundary condition which precluded the existence of any non-trivial unstable perturbations. Thus, as far as stability is concerned, the limiting case $(d / D)=\infty$ corresponding to the Impinging of a finite jet on a plate of extremely large width does not correspond to the case of a jet impinging on a truly infinite wall. The latter problem is equivalent to that of perturbations which are symmetric about the vertical axis of two equal and opposite jets impinging upon each other. It is considered in Section 4.

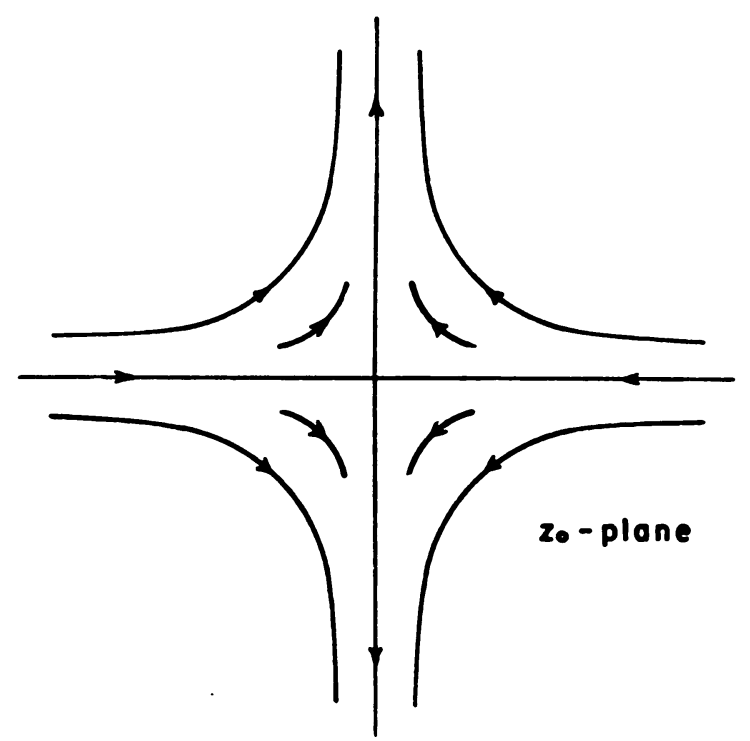

FIG. 3.

4. Equal and opposite jets. In this section we shall consider the flow of two equal and opposite two-dimensional jets which impinge upon each other (Fig. 3).

The potential of the basic flow in the hodograph plane is readily found as that due 
to two sources at $w= \pm 1$ and two sinks at $w= \pm i$, all of the same strength (Fig. 4).

The flow possesses a vertical axis of symmetry as well as a horizontal one and the perturbation problem is conveniently divided into two problems, that of perturbations symmetric and antisymmetric about the vertical axis of symmetry. These correspond in term to demanding that $w_{2} / w$ be either real or purely imaginary, respectively, on the imaginary axis in the hodograph plane.

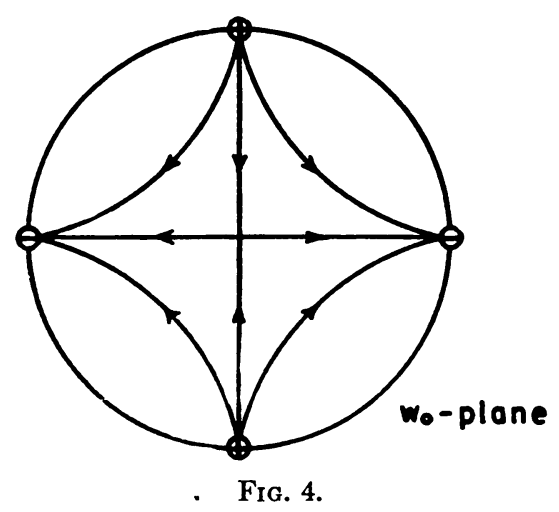

Now if one proceeds as in Section 3 to consider the boundary conditions on $w_{2} / w$ mentioned above along with analyticity and symmetry requirements and the free surface condition, one is again led to perturbations which are solutions of an inhomogeneous differential equation. In terms of a new variable $\zeta=w^{2}$ the equation can be reduced to a standard form known as Heun's equation [10] and [11] possessing regular singular points at $\zeta=0,1,-1$, and $\infty$.

Again proceeding as in Section 3 we can apply the remaining physical boundary conditions to the solutions of the perturbation equation. It is found that for perturbations which are symmetric about the vertical axis of symmetry, there exist non-trivial solutions with $0 \leq \operatorname{Re}\{\lambda\}<1$. All other admissible perturbations have $\operatorname{Re}\{\lambda\}<0$.

5. Generalized orifices. Each of the flows to be considered in this section represents the draining of an infinite reservoir through an orifice (Fig. 5). The sides of the orifice are made up of two semi-infinite planes inclined to each other at an angle of $2 \pi / n$ radians, where $n=2^{p}, p=0,1,2, \cdots$. When $p=0$ the configuration becomes the Borda mouthpiece which has been treated by Ablow and Hayes, and hence this section is essentially a generalization of that problem.

The similarity of these flows for various $n$ is seen by examining the potential of the basic flow in the hodograph plane. The flow in the physical plane is mapped into a sector of the unit circle in the hodograph plane bounded by radii inclined at an angle of $\pm \pi / n$ radians to the positive real axis (Fig. 6).

To find the potential for which the circular arc and radii are streamlines, we image the sector to cover the entire unit circle. The basic flow potentials for all $n$ are similar in that they may be thought of as arising from the presence of a source at the origin and sinks on the unit circle at the $n$ roots of unity. Thus, the potential may be written as

$$
f_{0}(w)=\log \frac{w^{n / 2}}{\left(w^{n}-1\right)^{2}} .
$$




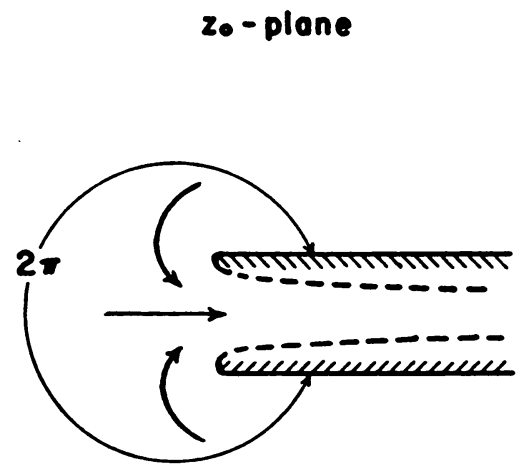

(o) $n=1$



(b) $n=2$

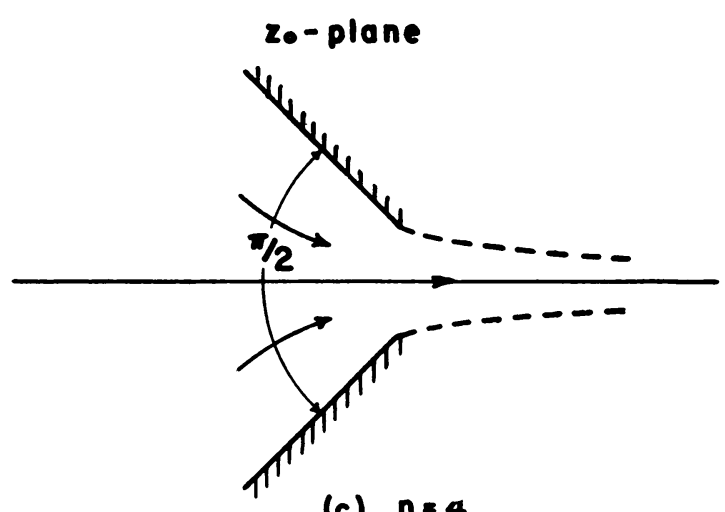

(c) $n=4$

FIG. 5.

But if we transform to a new variable $\zeta=w^{n}$ the potential becomes

$$
f_{0}(\zeta)=\frac{1}{2} \log \frac{\zeta}{(\zeta-1)^{2}}
$$

which provides a single representation for the potential of all the flows considered here. We are now in a position to investigage the stability of the flows for all $n$ by a single analysis.

In this problem the wall streamline and free surface conditions lead to an inhomogeneous second order differential equation governing the perturbations which can be put into the standard form of the hypergeometric equation having regular singular points at $\zeta=0,1$ and $\infty$.

After writing the solutions to the perturbation equation, the application of the boundary conditions, which are similar to those applied in Section 3 , shows that all admissible perturbations have $\operatorname{Re}\{\lambda\}<0$. One difference noted here is that it appears possible to satisfy all boundary conditions with an antisymmetric perturbation made 


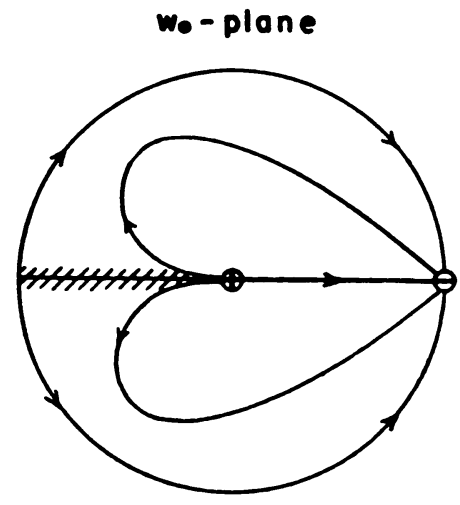

(o) $n=1$



(b) $n=2$

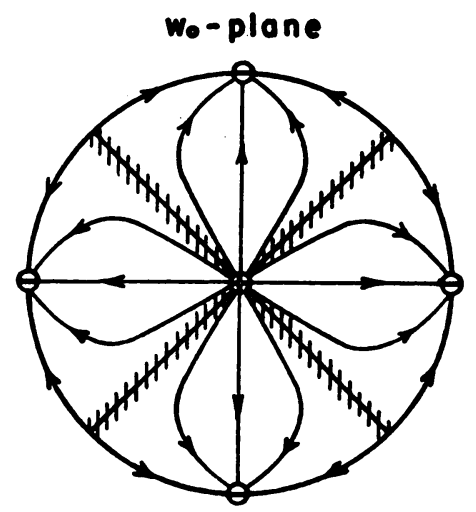

(c) $n=4$

Fig. 6.

up only of terms from the complementary solutions. This is possible if $\lambda$ takes on the values

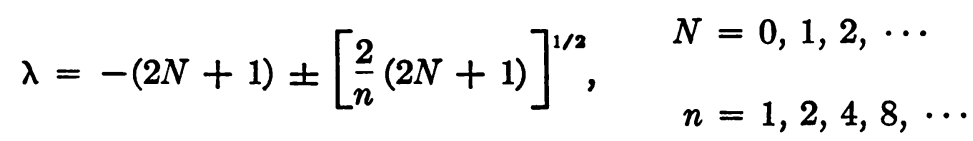

We note that for $N=0$ and $n=1, \lambda=-1+2^{1}$ which has a positive real part.

Thus, for all $n$ but $n=1$ we have only stable perturbations, while for $n=1$, corresponding to the Borda mouthpiece flow, there exists an isolated unstable perturbation.

6. Hollow vertex bounded by cylindrical walls. This problem is a generalization of a problem treated by Ablow and Hayes who considered the unbounded flow about a hollow vortex.

The basic flow is a cyclic irrotational motion with circular streamlines bounded on the outside by a solid circular wall and on the inside by a concentric circular hollow 
vortex forming a constant pressure surface. The basic flow potential in the hodograph plane is simply

$$
f_{0}(w)=i \log w .
$$

This flow has an antisymmetric velocity distribution and hence we may no longer decompose an arbitrary perturbation into symmetric and antisymmetric components which satisfy the boundary conditions separately.

Due to the simplicity of the basic flow potential, however, one can assume the form of the perturbation potential as

$$
f_{2}=G_{1}(w) e^{\lambda t}+G_{2}(w) e^{\lambda * t},
$$

where the forms for $G_{1}$ and $G_{2}$ may be deduced from a knowledge of the basic flow behavior as

$$
\begin{aligned}
& G_{1}(w)=B_{1} \log (w)+\sum_{-\infty}^{\infty} a_{r} w^{r}, \\
& G_{2}(w)=B_{2} \log (w)+\sum_{-\infty}^{\infty} b_{r} w^{r},
\end{aligned}
$$

with $B_{1}, B_{2}, a_{r}$ and $b_{r}$ arbitrary constants.

Applying the boundary conditions that the circular wall remains a streamline and that the perturbation satisfies the free surface condition (2.17) we find an infinite set of eigenvalues

$$
\lambda_{n}=-i\left(n \pm[N(n)]^{1 / 2}\right)
$$

for all $n$, where

$$
N(n)=n\left[\frac{1-\alpha^{2 n}}{1+\alpha^{2 n}}\right]
$$

and $\alpha$ is the ratio of the radius of the hollow vortex to that of the wall; $\lambda_{n}$ is purely imaginary and we conclude that the basic flow is a neutrally stable configuration.

Corresponding to each value of $n$ we can now find an elementary perturbation $f_{2 n}$ and can generate any admissible perturbation by summing the elementary solutions for all $n$. With $f_{2}$ known we can evaluate the perturbation of the free surface from (2.10). Aside from the arbitrariness introduced by the function $\chi$ the perturbation is made up of a wave pattern whose components travel at angular velocities equal to $1 \pm[N(n)]^{1 / 2} / n$, i.e., they either lead or lag the basic flow by an angular velocity of $[N(n)]^{\frac{3}{3}} / n$.

An interesting analogy with the propagation of gravity waves in water is found when the ratio of the depth of fluid between the vortex surface and the walls, to the radius of the vortex surface is made very small. At the same time we consider a fixed but arbitrary ratio of depth of fluid to wave length $\gamma$ of the disturbance. The propagation velocity of the disturbance is found to be approximately

$$
c^{2}=\frac{\gamma}{2 \pi a} \tanh \frac{2 \pi h}{\gamma}
$$

where $h$ is the depth of fluid between the free surface and the wall. This is identical with the velocity of the propagation of a gravity wave in water if $(1 / a)$ is replaced by the 
acceleration of gravity $g$. The term $(1 / a)$ represents approximately the centrifugal acceleration throughout the fluid for the case of small depth.

This problem has also been solved by Lord Kelvin in a different manner and after a change of notation the results presented here are seen to agree in detail with those found hy Kelvin.

\section{REFERENCES}

1. C. M. Ablow and W. I). Hayes, Perturbation of free surface flows, Technical Report No. 1, Office of Naval Research, Contract N7onr-35807, Graduate Division of Applied Mathematics, Brown University (1951).

2. H. Helmholtz, On the discontinuous movements of fluids, Phil. Mag., 36, 337 (1868).

3. G. Kirchoff, Zur Theorie freier Flüssigkeitsstrahlen, J. reine u. angew. Math., 70, 289 (1869).

4. L: M. Milne-Thomson, Theoretical hydrodynamics, Macmillan, second edition, 1950.

5. William Thomson, Vibrations of a columnar vortex, Phil. Mag. (5) 10, 155 (1880).

6. H. Lamb, Hydrodynamics, Dover Publications, 6th edition, 1945.

7. A. Forsyth, Theory of differential equations, Part III, vol. 4, Cambridge University Press, 1902.

8. E. L. Ince, Ordinary differential equations, Dover Publications.

9. $\mathrm{K}$. W. Mangler, Improper integrals in theoretical aerodynamics, Royal Aircraft Establishment, Farnborough, Report Aero. 2424 (1951).

10. K. Heun, Zur Theorie der Riemann'schen Functionen zweiter Ordnung mit vier Verzweigungspunkten, Math. Ann. 33, 161 (1889).

11. C. Snow, The hypergeometric and Legendre functions with application to integral equations of potential theory, National Bureau of Standards (1942). 